\title{
Erythema ab igne: A case report
}

\author{
Yogesh D ${ }^{1}$, B.D. Sathyanarayana ${ }^{2}$, M.R. Swaroop ${ }^{3}$, Sindhujaa Sreekanth $\mathrm{S}^{4}$, Shruti Bidarkar ${ }^{5, *}$ \\ ${ }^{1}$ Assistant Professor, ${ }^{2}$ Professor and HOD, ${ }^{3}$ Associate Professor, ${ }^{4,5}$ Junior Resident, Dept. of Dermatology, Rajiv Gandhi \\ University of Health Sciences, Karnataka, India
}

*Corresponding Author:

Email: shrutibidarkar27@gmail.com

\begin{abstract}
Erythema ab igne is an asymptomatic cutaneous condition characterized by reticular, erythematous to violaceous patches on sites of exposure to heat. It is a rare condition but its incidence has been rising as heating sources are being used to treat chronic pain. We report a case of erythema ab igne over the lower back in a 23 -year-old girl following the use of heat pads for back ache.
\end{abstract}

Keywords: Erythema ab igne, Heating pads, Asymptomatic, Reticulate.

\section{Introduction}

Erythema ab igne (EAI) or erythema caloricum is an acquired dermatoses caused by repeated and prolonged exposure of the skin to heat or thermal radiation. EAI is known to be a classic example of environmental and occupational dermatosis. It presents as reticular, erythematous to violaceous patches on sites of exposure to heat. Although it can present on any site, most cases of erythema ab igne are on the back or thighs, correlating with areas where heating pads are commonly used. Erythema ab igne can resemble numerous skin conditions including livedo reticularis, livedo racemosa, cutis marmorata, and cutis marmorata telangiectasia. Diagnosis is mainly based on history and clinical examination. Histopathological examination shows non-specific findings ranging from a sparse perivascular infiltrate in early lesions to epidermal atrophy, telangiectasias, keratinocyte atypia, and hemosiderin deposition in late stages. Treatment involves avoiding further exposure to heat source and use of heat therapy intermittently. Cases of erythema $a b$ igne may prove to be diagnostically challenging due to lack of familiarity with the condition. While this dermatosis carries a favorable prognosis, nonmelanoma skin cancers have been reported to arise within the lesions of erythema ab igne. Hence these patients have to be carefully followed up.

\section{Case Report}

A 23-year-old girl presented with asymptomatic red to brown flat lesions over the lower back since two weeks. She had a history of continuous low backache since one and half months for which she had been advised treatment with oral medications along with heating pads as hot fomentation and physiotherapy with short wave diathermy. She gave history of usage of hot pads for backache every now and then for the last few months. There was no itching or burning sensation over the area. There was no history of any raised skin lesions prior. There were no similar complaints elsewhere on the body. On examination, few erythematous macules and patches in reticulate pattern and multiple reticulate hyperpigmented macules and patches were found on lower back extending from $\mathrm{T} 8$ dermatome down up to sacral region. There was also mild surface atrophy. Based on the history and clinical examination, a diagnosis of EAI was made. Dermoscopy showed Arcuate pattern, Dense pigment network and Reddish hue. Patient was given moisturizers to be applied over the patches and was counselled about the benign nature of the condition. She was advised to restrict the use of hot pads over the back. During the heat therapy sessions, we recommended her to use heating pads in 15-20-minute intervals and not to exceed 30 minutes of use in a single therapeutic session. She was also advised that the pad be placed on top of the affected area, and not underneath. Due to a potential risk of malignancy, patient was advised regular follow up.

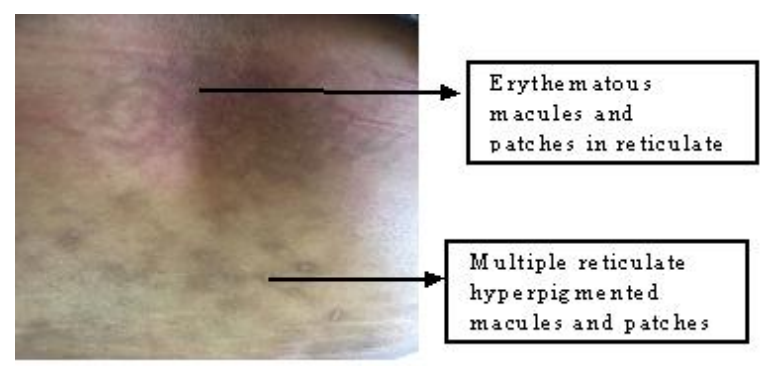

Fig. 1:

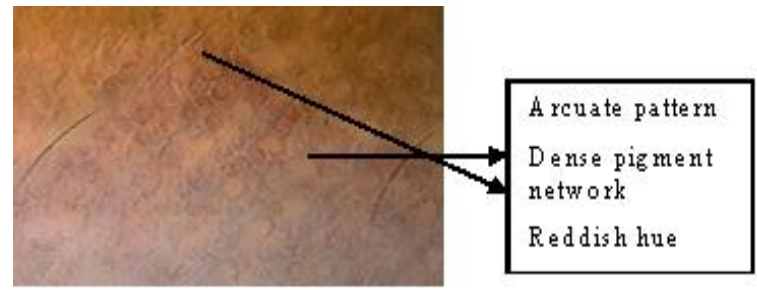

Fig. 2:

\section{Discussion}

Erythema ab igne presents initially as a transient, blanchable erythema followed by progression to a reticular pattern of hyperpigmentation with epidermal atrophy and telangiectasias after continued heat 
exposure. ${ }^{1}$ The incidence of EAI in India is variable. ${ }^{2}$ It is found to be detected in the range of 43-47 degree celsius during which the heat exposure results in cutaneous hyperthermia. ${ }^{3}$ As erythema ab igne is caused by prolonged exposure to heat sources at temperatures insufficient to cause burns (between 37 and $\left.113^{\circ} \mathrm{F}\right)$, it is typically asymptomatic. ${ }^{3}$ Heat radiation may be due to repeated body-warming techniques like the use of Kangri by people from northern India, ${ }^{4}$ frequent hot bathing, ${ }^{5}$ hot water bottles, heating pads and cooking. Seasonal incidence of EAI occurs due to frequent exposure to heat radiation in order to avoid cold weather. ${ }^{2}$ EAI poses an occupational threat for silver smiths, jewellers, bakers and kitchen workers. ${ }^{2}$ It clinically presents as reticular, pigmented dermatoses and rarely may also present as bullous lesions. ${ }^{4}$ The distribution of EAI depends on the occupation, source of heat, the direction of the incident radiation, skin type and interposition of the clothing. ${ }^{1,2}$ Though erythema ab igne was believed to be associated with exposure to open fires and wood-burning stoves, the condition is now related to more technologically advanced sources of heat such as heating pads, space heaters, heated car seats, and laptop computers and also physiotherapy short wave diathermy. ${ }^{5}$ In our patient, the lesions were observed on the back after usage of heating pads and short wave diathermy for low backache. Given the asymptomatic nature and frequent difficulty in visualizing the site (especially when on the back), patients may be unaware that they have developed this hyperpigmented dermatosis. EAI should be differentiated from vasculitis, livedo reticularis, ${ }^{1}$ port wine stain, cutis marmorata and poikiloderma.

Skin biopsies may demonstrate a range of findings, from a sparse perivascular infiltrate in early lesions to epidermal atrophy, telangiectasias, keratinocyte atypia, and hemosiderin deposition. Late, well-established lesions can demonstrate increased elastin fibers, which may require special stains to identify. ${ }^{4}$ As histopathologic findings are generally nonspecific, skin biopsy is more helpful to exclude other differential diagnoses (such as cutaneous vasculitis) rather than to confirm a diagnosis of erythema ab igne.

The most effective treatment measure is immediate removal of the heat source and the lesions clear spontaneously within several weeks to months. While erythema $a b$ igne carries a favorable prognosis, squamous cell carcinoma and Merkel cell carcinoma have been reported to arise from these lesions. ${ }^{2,4}$ EAI has also been reported in patients with internal malignancy. ${ }^{6}$

If lesions of erythema ab igne continue to evolve or ulcerate, biopsy should be performed to rule out malignancy. Prevention can occur both primarily with patient education and secondarily by early detection and removal of a causative heat source. Physicians recommending the use of heating pads should be aware of associated adverse cutaneous events and should counsel patients on safe practice while using these products. Proper use of heating pads should also be part of nursing and physician orders when this mode of pain relief is utilized in the inpatient setting. Safe practice guidelines recommend that, the heating pads are to be used in 15-20-minute intervals and to never exceed 30 minutes of use in a single therapeutic session ${ }^{6}$. Recommendations also include that the pad be placed on top of, and not underneath a body part, as trapped heat can increase temperature and in turn increase the risk of development of erythema ab igne. ${ }^{6}$

Erythema ab igne is an under recognised entity. Clinicians especially physiotherapist should be aware of this condition and provide education regarding appropriate and safe use of heating devices to patients requiring this mode of treatment.

\section{References}

1. Gaikwad KA, Khedker MY, Ban AP. Erythema ab igne. Indian J Dermatol Venereol Leprol, 1990;56:119-20.

2. Basavaraj KH, Kanthraj GR, Shetty AM, Rangappa V. Erythema ab igne in a rural Indian woman. Indian J Dermatol Venereol Leprol, 2011;77:731.

3. Bimbi C, Brzezinski P. Laptop-induced Erythema ab Igne - a case report. Our Dermatol Online, 2014;5(4):430-1.

4. Kesty $\mathrm{K}$ and Feldman SR. Erythema ab igne: evolving technology, evolving presentation Dermatology Online Journal 2014;20:10.

5. Dovretzky I and Silverman NR. Reticularerythema of the lower back. Arch Dermatol 1991;127:405-9.

6. Kuwahara RT, Carter S. A netlike rash. West J Med 2000;173(6):370-1. 